Some Theorems on the Expressive Limitations of Modal Languages

Author(s): Harold T. Hodes

Source: Journal of Philosophical Logic, Feb., 1984, Vol. 13, No. 1 (Feb., 1984), pp. 13-26

Published by: Springer

Stable URL: https://www.jstor.org/stable/30226293

JSTOR is a not-for-profit service that helps scholars, researchers, and students discover, use, and build upon a wide range of content in a trusted digital archive. We use information technology and tools to increase productivity and facilitate new forms of scholarship. For more information about JSTOR, please contact support@jstor.org.

Your use of the JSTOR archive indicates your acceptance of the Terms \& Conditions of Use, available at https://about.jstor.org/terms 
HAROLD T. HODES

\section{SOME THEOREMS ON THE EXPRESSIVE \\ LIMITATIONS OF MODAL LANGUAGES}

In [3] Allen Hazen considers certain statements expressible in an extensional language involving quantification over possible worlds, but which are not expressible in the corresponding modal language. In this paper, I derive a derive a variety of such claims from several model-theoretic theorems concerning S5.

\section{PRELIMINARIES}

Fix a set of predicates Pred, each of a particular number of places, and a countably infinite set of variables $\mathrm{Var}$; for a set of names $C$ form the language $L(C)$ by following the usual formation rules, with the primitives ' $L$ ', ' $\supset$ ', ' $\forall$ ' and ' $\square$ '. A term of $L(C)$ is a member of $\operatorname{Var} \cup C$. Use and mention shall be freely confused. Let $\urcorner \phi,(\exists \nu) \phi, \diamond \phi$ and $E \sigma$ abbreviate $\phi \in \perp\urcorner,(\forall \nu)\urcorner \phi,\urcorner \square\urcorner \phi$ and $(\exists \nu)(\nu=\sigma)$ where $\sigma$ is a term of $L(C)$ and $\nu$ is a variable distinct from $\sigma$; ' $\&$ ', ' $\vee$ ' and $` \equiv$ ' are defined as usual.

We work entirely within the modal logic S5. So we may take a frame to be a pair $F=(W, A), W$ a non-empty set, $A$ a function on $W$ so that $A(w)$ is a set for all $w \in W$, and $\cup\{A(w) \mid w \in W\}=\bar{A}$ is non-empty. An $F$-valuation for $L(C)$ is a function $V$ with domain $C \cup(W \times$ Pred $), V(c) \in \bar{A}$ for $c \in C$; for $w \in W$ and $P \in$ Pred, $P$-place: if $n \geqslant 1, V(w, P) \subseteq \bar{A}^{n}$; for $n=0$, $V(w, P) \in\{t, f\}$. A structure for $L(C)$ is a triple $\mathscr{A}=(W, A, V), V$ a $(W, A)$. valuation for $L(C)$. For $w \in W$, we say that $w$ is from $\mathscr{A} ; \bar{a}$ is an assignment for $\mathscr{A}$ iff $\bar{a}: \operatorname{Var} \rightarrow \bar{A}$. Let:

$$
\operatorname{den}(\mathscr{A}, \bar{a}, \sigma)= \begin{cases}\bar{a}(\sigma) & \text { if } \sigma \in \mathrm{Var} \\ V(\sigma) & \text { if } c \in C .\end{cases}
$$

Define $(\mathscr{A}, w)=\phi[\bar{a}]$, “ $\bar{a}$ satisfies $\phi$ at $w$ in $\mathscr{A}$ ", by the familiar recursion:

$$
\begin{aligned}
& (\mathscr{A}, w) \not \perp[\bar{a}] ; \\
& (\mathscr{A}, w) \vDash P[\bar{a}] \text { iff } V(w, P)=t \text { for } P 0 \text {-place; }
\end{aligned}
$$

Journal of Philosophical Logic 13 (1984) 13-26. 0022-3611/84/0131-0013\$01.40. ○ 1984 by D. Reidel Publishing Company. 


$$
\begin{aligned}
& (\mathscr{A}, w) \models P \sigma_{1} \ldots \sigma_{n}[\bar{a}] \text { iff }\left(a_{1}, \ldots, a_{n}\right) \in V(w, P) \text { where } \\
& a_{i}=\operatorname{den}\left(\mathscr{A}, \bar{a}, \sigma_{i}\right) \text { for } 1 \leqslant i \leqslant n \text { and } P \text { is } n \text {-place, } n \geqslant 1 ; \\
& (\mathscr{A}, w) \vDash \sigma=\sigma^{\prime}[\bar{a}] \text { iff } \operatorname{den}(\mathscr{A}, \bar{a}, \sigma)=\operatorname{den}\left(\mathscr{A}, \bar{a}, \sigma^{\prime}\right) ; \\
& (\mathscr{A}, w) \vDash(\phi \supset \psi)[\bar{a}] \text { if }(\mathscr{A}, w) \not \phi[\bar{a}] \text { or }(\mathscr{A}, w) \vDash \psi[\bar{a}] \text {; } \\
& (\mathscr{A}, w) \vDash(\forall \nu) \phi[\bar{a}] \text { iff for every } a \in A(w),(\mathscr{A}, w) \vDash \phi\left[\bar{a}_{a}^{\nu}\right] \\
& \text { where } \bar{a}_{a}^{\nu} \text { is the assignment which differs from } \bar{a} \text { at most in } \\
& \text { that it sends } \nu \text { to } a ;
\end{aligned}
$$

$$
(\mathscr{A}, w) \models \square \phi[\bar{a}] \text { iff for every } w^{\prime} \in W,(\mathscr{A}, w) \vDash \phi[\bar{a}] .
$$

Where $\Gamma$ is a set of formulae, $(\mathscr{A}, w) \vDash \Gamma[\bar{a}]$ iff for all $\phi \in \Gamma,(\mathscr{A}, w) \vDash$ $\phi[\bar{a}]$. Where the variables free in $\phi$ are understood to be $x_{1}, \ldots, x_{n}$, we'll write " $\left[a_{1}, \ldots, a_{n}\right]$ " instead of "[ $\left.\bar{a}\right]$ ", where it's understood that $a_{i}=\bar{a}\left(x_{i}\right)$ for $1 \leqslant i \leqslant n$. Let $(\mathscr{A}, w) \vDash \phi$, " $\phi$ is true at $w$ in $\mathscr{A}$ ", iff for all assignments $\bar{a}$ from $\mathscr{A},(\mathscr{A}, w) \models \phi[\bar{a}]$; note: free variables in $\phi$ are thus treated as if bound by a "possibilist" universal quantifier, not by ' $\forall$ '. $(\mathscr{A}, w) \vDash \Gamma$ is defined similarly. Where $\Gamma \cup\{\phi\}$ is a set of sentences, $\Gamma$ implies $\phi$ iff for all structures $\mathscr{A}, w$ from $\mathscr{A}$ and assignments $\bar{a}$ for $\mathscr{A}$, if $\mathscr{A}=\Gamma[\bar{a}]$ then $\mathscr{A} \vDash \phi[\bar{a}] . \phi$ is valid iff the empty set implies $\phi$.

We formalize quantified $\mathrm{S} 5$ as in [2]. Keep in mind that the axiom schema of universal instantiation is: $(\forall \nu) \phi \supset(E \sigma \supset \phi(\nu / \sigma))$ where $\sigma$ is a term of $L(C)$ substitutable for $\nu$ in $\phi$. Other axiom schemata worth keeping in mind are $(\forall \nu) E \nu$ and $\diamond E \sigma$ (for all terms $\sigma$ of $L(C)$ ). Our rules of proof are Modus Ponens, Universal Generalization and Necessitation. Where $\Gamma \cup\{\phi\}$ is a set of formulae, $\Gamma \vdash \phi$ iff $\vdash \phi$ or $\vdash\left(\psi_{1} \& \ldots \& \psi_{n}\right) \supset \phi$ for some $\psi_{1}, \ldots, \psi_{n} \in \Gamma$. Let $\diamond \Gamma=\left\{\diamond\left(\psi_{1} \& \ldots \& \psi_{n}\right) \mid \psi_{1}, \ldots, \psi_{n} \in \Gamma\right\}$.

A diagram over $W, C$ is a set of ordered pairs $(w, \phi), w \in W$ and $\phi$ a sentence of $L(C)$. Where $D$ is a diagram, $D(w)=\{\phi \mid(w, \phi) \in D\} ; \diamond D=$ $\cup\{\diamond D(w) \mid w \in W\} ;$ for $W^{\prime} \subseteq W, D^{-W^{\prime}}=D-\left\{(w, \phi) \mid w \in W^{\prime}\right\} ; D$ is consistent iff $\diamond D$ is consistent. We note the following facts.

If $D$ is consistent, then so is either $D \cup\{w, \phi)\}$ or $D \cup\{w, \neg \phi)\}$.

If $D \cup\{(w,(\exists v) \phi)\}$ is consistent and $c \in C$ does not occur in $D \cup\{(w,(\exists v) \phi)\}$ then $D \cup\{(w,(\exists \nu) \phi),(w, \phi(\nu / c))$, $(w, E c)\}$ is consistent. 
(3)

$$
\begin{gathered}
\text { If } D \cup\{(w, \diamond \phi)\} \text { is consistent and } w^{\prime} \in W \text { does not occur in } \\
\left.D \cup\{(w, \diamond \phi)\} \text { then } D \cup\{w, \diamond \phi),\left(w^{\prime}, \phi\right)\right\} \text { is consistent. }
\end{gathered}
$$

\section{THREE HENKIN COSTRUCTIONS}

We consider a language $L\left(C_{0}\right)$. Let $T_{0}$ be the set of universal closures of all formulae of the form:

$$
\phi \supset \square\left(\forall x_{1}\right) \square \ldots \square\left(\forall x_{n}\right) \diamond\left(\phi \& E x_{1} \& \ldots \& E x_{n}\right),
$$

where $\phi$ is a formula of $L\left(C_{0}\right)$ in which $x_{1}, \ldots, x_{n}$ are not free.

THEOREM 1. Let $T$ be a set of sentences of $L\left(C_{0}\right) . T \cup T_{0}$ is consistent iff there is a structure $\mathscr{A}=(W, A, V)$ for $L\left(C_{0}\right)$ and a $w_{0} \in W$ so that $\left(\mathscr{A}, w_{0}\right)=T$ and $\bar{A}=A\left(w_{0}\right)$.

Suppose $\mathscr{A}=(W, A, V)$ and $A\left(w_{0}\right)=\bar{A}$; then $\left(\mathscr{A}, w_{0}\right) \vDash T_{0}$. This proves the "if" direction. We now consider the "only if" direction. Note that $T_{0} \vdash(\exists x) E x$ and $T_{0} \vdash E c$ for all $c \in C_{0}$. Suppose $T \cup T_{0}$ is consistent. Then so is $\Gamma=T \cup T_{0} \cup\{(\exists x) E x\} \cup\left\{E c \mid c \in C_{0}\right\}$. Select sets $W, C \supseteq C_{0}$, $\operatorname{card}(W)=\operatorname{card}\left(C-C_{0}\right)=\max \left\{\aleph_{0}, \operatorname{card}(\operatorname{Pred}), \operatorname{card}\left(C_{0}\right)\right\}=\kappa$, Fix a listing in order-type $\kappa$ of all pairs $(w, \phi), w \in W$ and $\phi$ a sentence of $L(C)$; select $w_{0} \in W$ and let $D_{0}=\left\{w_{0}\right\} \times \Gamma . D_{0}$ is a consistent diagram. We now construct a sequence $\left\{D_{\xi}\right\}_{\xi \leqslant \kappa}$ of consistent diagrams. Suppose $D_{\xi}$ has been defined, is consistent, and for every $c \in C$ occurring in $D_{\xi}, E c \in D_{\xi}\left(w_{0}\right)$. Let $(w, \phi)$ be the $\xi$ th pair on our list.

If all names occurring in $\phi$ already occur in $D_{\xi}$, let:

$$
D_{\xi}^{\prime}= \begin{cases}\left.D_{\xi} \cup\{w, \phi)\right\} & \text { if this is consistent; } \\ \left.\left.D_{\xi} \cup\{w,\urcorner \phi\right)\right\} & \text { otherwise }\end{cases}
$$

By remark (1), $D_{\xi}^{\prime}$ is consistent. Otherwise suppose $c_{1}, \ldots, c_{n}$ are the members of $C$ occurring in $\phi$ but not in $D_{\xi}$. Let

$$
D_{\xi}^{\prime}= \begin{cases}\left.D_{\xi} \cup\{w, \phi),\left(w_{0}, E c_{1}\right), \ldots,\left(w_{0}, E c_{n}\right)\right\} & \text { if this is consistent } \\ \left.D_{\xi} \cup\{w, \neg \phi),\left(w_{0}, E c_{1}\right), \ldots,\left(w_{0}, E c_{n}\right)\right\} & \text { otherwise. }\end{cases}
$$

Suppose both $D_{\xi} \cup\left\{(w, \phi),\left(w_{0}, E c_{1}\right), \ldots\right\}$ and $D_{\xi} \cup\left\{(w, \neg \phi),\left(w_{0}, E c_{1}\right)\right.$, $\ldots\}$ are inconsistent. Suppose $w \neq w_{0}$. For some $\theta$, a conjunction of mem-

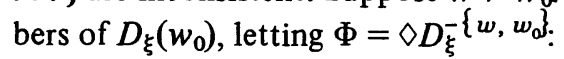




$$
\begin{aligned}
& \left.\Phi \cup \diamond\left(D_{\xi}(w) \cup\{\phi\}\right) \vdash \square\left(\theta \supset\left(\neg E c_{1} \vee \ldots \vee\right\urcorner E c_{n}\right)\right), \\
& \Phi \cup \diamond\left(D_{\xi}(w) \cup\{\neg \phi\} \vdash \square\left(\theta \supset\left(\neg E c_{1} \vee \ldots \vee \neg E c_{n}\right)\right) .\right.
\end{aligned}
$$

By induction hypothesis, for the $c \in C$ occurring in $\theta$ we have $E c \in D_{\xi}\left(w_{0}\right)$; since $T_{0} \subseteq D_{\xi}\left(w_{0}\right)$,

$$
D_{\xi}\left(w_{0}\right) \vdash \square\left(\forall x_{1}\right) \square \ldots \square\left(\forall x_{n}\right) \diamond\left(\theta \& E x_{1} \& \ldots \& E x_{n}\right) .
$$

Since $\vdash \diamond E c_{i}$ for $i=1, \ldots, n$,

$$
D_{\xi}\left(w_{0}\right) \vdash \diamond\left(\theta \& E c_{1} \& \ldots \& E c_{n}\right) \text {. }
$$

From this, using S5 axioms, we may show that $D_{\xi} \cup\{(w, \phi)\}$ and $D_{\xi} \cup$ $\{(w,\urcorner \phi)\}$ are both inconsistent, contrary to the consistency of $D_{\xi}$. Now suppose $w=w_{0}$. For $\theta$, a conjunction of members of $D_{\xi}\left(w_{0}\right)$,

$$
\begin{aligned}
& \diamond D_{\xi}^{-\left\{w_{0}\right\}} \vdash \square\left((\theta \&(\exists x) E x \& \phi) \supset\left(\neg E c_{1} \vee \ldots \vee \neg E c_{n}\right)\right), \\
& \diamond D_{\xi}^{-\left\{w_{0}\right\}} \vdash \square\left((\theta \&(\exists x) E x \& \neg \phi) \supset\left(\neg E c_{1} \vee \ldots \vee \neg E c_{n}\right)\right)
\end{aligned}
$$

Thus

$$
\diamond D_{\xi}^{\left\{w_{0}\right\}} \vdash(\theta \&(\exists x) E x) \supset\left(\neg E c_{1} \vee \ldots \vee \neg E c_{n}\right) .
$$

But since $c_{1}, \ldots, c_{n}$ do not occur in $D_{\xi}^{-\left\{w_{\omega}\right\}}$ or $\theta$, they may be replaced by variable and universally quantified; then by standard quantifier manipulations,

$$
\diamond D_{\xi}^{-\left\{w_{0}\right\}} \vdash(\theta \&(\exists x) E x) \supset(\forall x) \neg E x .
$$

Therefore $\diamond D_{\xi}^{-\left\{w_{0}\right\}} \vdash \square า(\theta \&(\exists x) E x)$, showing that $D_{\xi}$ is inconsistent. Thus $D_{\xi}^{\prime}$ is consistent. Let $\phi^{\prime}$ be $\phi$ or $\urcorner \phi$ according to the case used in defining $D_{\xi}^{\prime}$. If $\phi^{\prime}$ is neither $(\exists \nu) \psi$ nor $\diamond \psi$, let $D_{\xi+1}=D^{\prime}$.

If $\phi^{\prime}$ is $(\exists \nu) \psi$, select a $c \in C$ not occuring in $D_{\xi}^{\prime}$ and let $D_{\xi}^{\prime \prime}=D_{\xi}^{\prime} \cup$ $\{(w, \psi(\nu / c)),(w, E c)\} ; D_{\xi}^{\prime \prime}$ is consistent. Let $D_{\xi+1}=D_{\xi}^{\prime \prime} \cup\left\{\left(w_{0}, E c\right)\right)$. If $D_{\xi+1}$ were inconsistent there would be a $\theta$, a conjunction of members of $D_{\xi}^{\prime \prime}\left(w_{0}\right)$, so that $\diamond D_{\xi}^{\prime \prime}-\left\{w_{0}\right\} \vdash \square(\theta \supset \neg E c)$. But $D_{\xi}^{\prime \prime}\left(w_{0}\right) \vdash \square(\forall x) \diamond(\theta \& E x)$; so $D_{\xi}^{\prime \prime}\left(w_{0}\right) \vdash \diamond(\theta \& E c)$, contrary to the consistency of $D_{\xi}^{\prime \prime}$.

If $\phi^{\prime}$ is $\diamond \psi$, select a $w^{\prime} \in W$ not occurring in $D_{\xi}^{\prime}$ and let $D_{\xi+1}=$ $D_{\xi}^{\prime} \cup\left\{\left(w^{\prime}, \psi\right)\right\} . D_{\xi+1}$ is consistent.

For $\lambda \leqslant \kappa, \lambda$ a limit ordinal, $D_{\lambda}=\cup\left\{D_{\xi} \mid \xi<\lambda\right\}$. $D_{\lambda}$ is consistent; and for any $c \in C$ occurring in $D_{\lambda}, E c \in D_{\lambda}\left(w_{0}\right) . D_{K}$ is a maximal consistent diagram over $W$ and $C$. It determines a structure $\mathscr{A}=(W, A, V)$ as follows. For $c, c^{\prime} \in C$, let $c \sim c^{\prime}$ iff for some $w \in W, c=c^{\prime} \in D_{\kappa}(w)$; let $[c]$ be the 
〜-equivalence class of $c$; let $A(w)=\left\{[c] \mid E c \in D_{\kappa}(w)\right\} ;$ let $V(c)=[c]$; $V(w, P)=t$ iff $P \in D_{\kappa}(w)$ where $P$ is 0-place; $V(w, P)=\left\{\left(\left[c_{1}\right], \ldots\right.\right.$, $\left.\left.\left[c_{n}\right]\right) \mid P c_{1} \ldots c_{n} \in D_{K}(w)\right\}$ for $P n$-place and $n \geqslant 1$. As usual, for all sentences $\phi$ of $L(C),(\mathscr{A}, w) \vDash \phi$ iff $\phi \in D(w)$. $\mathscr{A}$ is as desired.

For any formula $\phi$, let ( $) \phi$ be $\square\left(\forall \nu_{1}\right) \square \ldots \square\left(\forall \nu_{n}\right) \square \phi$, if $\nu_{1}, \ldots, \nu_{n}$ are the variabes free in $\phi$; if $\phi$ is a sentence, ( $\square) \phi$ is $\square \phi$. Where $\Gamma$ is a set of formulae, (ㅁ) $\Gamma=\{(\square) \phi \mid \phi \in \Gamma\}$.

Let $T_{1}$ be the set of formulae of $L\left(C_{0}\right)$ of the form:

$$
(\diamond \phi \& \diamond \psi \& \square\urcorner(\phi \& \psi)) \supset \diamond(\phi \&(\exists x) \diamond(\psi \&\urcorner E x)),
$$

where $x$ is not free in $\psi$.

THEOREM 2. Suppose $T$ is a set of sentences of $L\left(C_{0}\right) . T \cup(\square) T_{1} \cup$ $\{\square(\exists x) E x\}$ is consistent iff there is a structure $\mathscr{A}=(W, A, V)$ for $L\left(C_{0}\right)$ and $w_{0} \in W$ such that $\left(\mathscr{A}, w_{0}\right) \mid=T$ and for all $w, w^{\prime} \in W$, if $w \neq w^{\prime}$ then $A(w)$ is not a subset of $A\left(w^{\prime}\right)$.

Suppose $\mathscr{A}=(W, A, V)$ is a structure for $L\left(C_{0}\right), w_{0} \in W$ and for all $w, w^{\prime} \in W$, if $w \neq w^{\prime}$ then $A(w)$ is not a subset of $A\left(w^{\prime}\right)$. Then $\left(\mathscr{A}, w_{0}\right) \vDash$ (ㅁ) $T_{1} \cup\{\square(\exists x) E x\}$. Clearly no $A(w)$ is empty. Suppose $\mathbf{a}=\left(a_{1}, \ldots, a_{n}\right)$, $a_{i} \in \bar{A}$ for $i=1, \ldots, n, w \in W$ and $\left.(\mathscr{A}, w) \vDash \diamond \phi \& \diamond \psi \& \square\right\urcorner(\phi \& \psi)[\mathrm{a}]$. Select $w_{1}, w_{2} \in W,\left(\mathscr{A}, w_{1}\right) \models \phi[\mathrm{a}],\left(\mathscr{A}, w_{2}\right) \vDash \psi[\mathrm{a}]$ and $w_{1} \neq w_{2}$; selecting $b \in A\left(w_{1}\right)-A\left(w_{2}\right)$ as a witness, $\left(\mathscr{A}, w_{1}\right) \vDash(\exists x) \diamond(\psi \& \neg E x)[\mathrm{a}]$. Thus $(\mathscr{A}, w)=\diamond(\phi \&(\exists x) \diamond(\psi \&\urcorner E x))[\mathrm{a}]$. So $\left(\mathscr{A}, w_{0}\right) \vDash(\square) T_{1}$. These remarks suffice to prove the "if" direction.

We now prove the "only if" direction. Fix $W, C$ and the sequence of pairs $(w, \phi)$ as in the previous argument. Let $D_{0}=\left\{w_{0}\right\} \times\left(T \cup(\square) T_{1} \cup\right.$ $\{(\exists x) E x\})$ for a selected $w_{0} \in W$. We construct a sequence of consistent diagrams under this constraint: only introduce a new world when consistency demands it. Furthermore, when we introduce a new world we also introduce new constants to insure that the final model has the desired structural property.

Suppose $D_{\xi}$ has been constructed, is consistent, and for all $w, w^{\prime} \in W$ and occurring in $D_{\xi}$, if $w \neq w^{\prime}$ then

$$
\diamond D_{\xi}^{-\{w, w\}} \cup \square\left(D_{\xi}(w) \cup D_{\xi}\left(w^{\prime}\right)\right) \text { is inconsistent; }
$$


(2)

$$
\begin{aligned}
& \text { There are } c, c^{\prime} \in C \text { so that } E c, \neg E c^{\prime} \in D_{\xi}(w) \text { and } \\
& E c^{\prime}, \neg E c \in D_{\xi}\left(w^{\prime}\right) \text {. }
\end{aligned}
$$

Let $(w, \phi)$ be the least pair in our list of pairs which has not been handled and for which $w$ occurs in $D_{\xi}$. Let

$$
D_{\xi}^{\prime}= \begin{cases}\left.D_{\xi} \cup\{w, \phi)\right\} & \text { if this is consistent; } \\ \left.\left.D_{\xi} \cup\{w,\urcorner \phi\right)\right\} & \text { otherwise. }\end{cases}
$$

Let $\phi^{\prime}$ be $\phi$ or $\urcorner \phi$ according to whether the first or second case applied. If $\phi^{\prime}$ is neither $(\exists \nu) \psi$ nor $\diamond \psi$, let $D_{\xi+1}=D_{\xi}^{\prime}$. The induction hypothesis is preserved. If $\phi^{\prime}$ is $(\exists \nu) \psi$, select $c \in C$ not occurring in $D_{\xi}^{\prime}$ and let $D_{\xi+1}=D_{\xi}^{\prime} \cup\{(w, \psi(\nu / c)),(w, E c)\}$. If $\phi^{\prime}$ is $\diamond \psi$ and for some $w^{\prime}$ occurring in $D_{\xi}^{\prime}, D_{\xi}^{\prime} \cup\left\{\left(w^{\prime}, \psi\right)\right\}$ is consistent, select such a $w^{\prime}$ and let $D_{\xi+1}=D_{\xi}^{\prime} \cup$ $\left\{\left(w^{\prime}, \psi\right)\right\}$. The induction hypothesis is preserved.

Suppose that there is no such $w^{\prime}$. Select a $w^{\prime} \in W$ not occurring in $D_{\xi}^{\prime}$ and let $D_{\xi}^{\prime \prime}=D_{\xi}^{\prime} \cup\left\{\left(w^{\prime}, \psi\right)\right\}$; this is consistent. Let $\left\{w_{\eta}\right\}_{\eta<\alpha}$ be a well-ordered list of the members of $W$ occurring in $D_{\xi}^{\prime}$. For each $\eta<\alpha$ select two members of $C$, call them $c_{\eta}$ and $d_{\eta}$, all distinct and not occurring in $D_{\xi}^{\prime \prime}$. Let $\left.\mathrm{D}_{\xi}^{\prime \prime \prime}=D_{\xi}^{\prime \prime} \cup\left\{w_{\eta}, E c_{\eta}\right),\left(w^{\prime}, E d_{\eta}\right) \mid \eta<\alpha\right\}$. Since $\square(\exists x) E x \in D_{\xi}\left(w_{0}\right)$ and all $c_{\eta}$ and $d_{\eta}$ are "new", $D_{\xi}^{\prime \prime \prime}$ is consistent. For $\eta \leqslant \alpha$, let:

$\left.D_{\xi, \eta}=D_{\xi}^{\prime \prime \prime} \cup\left\{\left(w_{\eta^{\prime}},\right\urcorner E d_{\eta^{\prime}}\right),\left(w^{\prime}, \neg E c_{\eta^{\prime}}\right) \mid \eta^{\prime}<\eta\right\}$. By induction on $\eta$, each $D_{\xi, \eta}$ is consistent. This holds for $\eta=0$. Suppose $D_{\xi, \eta}$ is consistent but

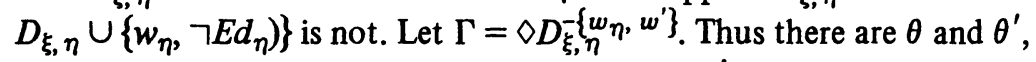
conjunctions of members of $D_{\xi, \eta}\left(w_{\eta}\right)$ and $D_{\xi, \eta}\left(w^{\prime}\right)$, so that

$$
\left.\Gamma \cup\left\{\diamond(\theta \&\urcorner E d_{\eta}\right), \diamond\left(\theta^{\prime} \& E d_{\eta}\right)\right\}
$$

is inconsistent. By (1) of our induction hypothesis we may, without loss of generality, suppose that $\Gamma \cup\left\{\diamond\left(\theta \& \theta^{\prime}\right)\right\}$ is inconsistent. Using the machinery of S5 we may conclude that

$$
\Gamma \vdash\left(\theta^{\prime} \& E d_{\eta}\right) \supset \square\left(\theta \supset E d_{\eta}\right) ;
$$

but since $d_{\eta}$ doesn't occur in $\Gamma, \theta$ or $\theta^{\prime}$,

$$
\Gamma \vdash \theta^{\prime} \supset(\forall x) \square(\theta \supset E x) .
$$

Because all members of $\Gamma$ begin with ' $\diamond$ ', using the machinery of S5:

$$
\Gamma \vdash \square\left(\theta^{\prime} \supset(\forall x) \square(\theta \supset E x)\right) .
$$

Because (口) $T_{1} \subseteq D_{\xi \eta}\left(w_{0}\right)$, 


$$
\left.\left.D_{\xi, \eta}\left(w_{0}\right) \vdash\left(\diamond \theta^{\prime} \& \diamond \theta \& \square\right\urcorner\left(\theta \& \theta^{\prime}\right)\right) \supset \diamond\left(\theta^{\prime} \&(\exists x)(\theta \&\urcorner E x\right)\right) .
$$

Since $\Gamma \vdash ロ\urcorner\left(\theta \& \theta^{\prime}\right)$,

$$
\diamond D_{\xi, \eta} \vdash \diamond\left(\theta^{\prime} \&(\exists x) \diamond(\theta \& \neg E x)\right),
$$

contrary to the consistency of $D_{\xi, \eta}$. Thus $D_{\xi, \eta} \cup\left\{\left(w_{\eta}, \neg E d_{\eta}\right)\right\}$ is consistent. A similar argument with $D_{\xi, \eta} \cup\left\{\left(w_{\eta}, \neg E d_{\eta}\right)\right\}$ in place of $D_{\xi, \eta}$, $c_{\eta}$ in place of $d_{\eta}$, and the roles of $w_{\eta}$ and $w^{\prime}$ exchanged, shows the consistency of $D_{\xi, \eta+1}$. Clearly $D_{\xi, \lambda}$ for $\lambda$ a limit ordinal $\leqslant \alpha$ is consistent. Thus $D_{\xi+1}=D_{\xi, \alpha}$ is consistent and satisfies our induction hypothesis. From $D_{\kappa}$ we construct the desired structure $\mathscr{A}=\left(W^{\prime}, A, V\right)$ as usual, with $W^{\prime}=$ $\left\{w B W \mid w\right.$ occurs in $\left.D_{k}\right\} . \mathscr{A}$ has the desired properties.

Q.E.D.

Let $T_{2}$ be the set of all formulae of $L\left(C_{0}\right)$ of the form:

$$
\psi \supset \square(\exists x) \diamond(\psi \& E x)),
$$

where $x$ is not free in $\psi$.

THEOREM 3. Suppose $T$ is a set of sentences of $L\left(C_{0}\right) . T \cup(\square) T_{2} \cup$ $\{\square(\exists x) E x\}$ is consistent iff there is a structure $\mathscr{A}=(W, A, V)$ and $w_{0} \in W$ so that $\left(\mathscr{A}, w_{0}\right) \equiv T$ and for all $w, w^{\prime} \in W, A(w)$ and $A\left(w^{\prime}\right)$ are not disjoint.

Clearly if $\mathscr{A}$ is as described on the right-hand side, $\left(\mathscr{A}, w_{0}\right) \vDash(\square) T_{2} \cup$ $\{\square(\exists x) E x\}$. The "if" direction follows.

Suppose $T \cup(\square) T_{2} \cup\{\square(\exists x) E x\}$ is consistent. Fix $W, C$ and the listing of ordered pairs $(w, \phi)$ as before; select $w_{0} \in W$ and let $D_{0}=\left\{w_{0}\right\} \times$ $\left(T \cup(\square) T_{2} \cup\{\square(\exists x) E x\}\right)$. We construct a sequence of consistent diagrams $\left\{D_{\xi}\right)_{\xi \leqslant \kappa}$ so that: for every $w, w^{\prime}$ occurring in $D$ there is a $c \in C$ so that $E c \in D(w)$ and $E c \in D\left(w^{\prime}\right)$. The construction is easy and therefore left to the reader.

We note the following corollaries to the previous theorems.

COROLLARY 1. $T_{0} \vdash \phi$ iff for all structures $\mathscr{A}=(W, A, V)$ for $L\left(C_{0}\right)$ and $w_{0} \in W$, if $\bar{A}=A\left(w_{0}\right)$ then $\left(\mathscr{A}, w_{0}\right) \vDash \phi . T_{0} \vdash \square \phi$ iff for all structures $\mathscr{A}=(W, A, V)$ for $L\left(C_{0}\right)$, if $\bar{A}=A\left(w_{0}\right)$ then $(\mathscr{A}, w) \vDash \phi$ for all $w \in W$.

COROLLARY 2. (ㅁ) $T_{1} \cup\{\square(\exists x) E x\} \vdash \phi$ iff for all structures $\mathscr{A}=(W, A, V)$ for $L\left(C_{0}\right)$ such that for all $w, w^{\prime} \in W$ if $w \neq w^{\prime}$ then $A(w) \notin A\left(w^{\prime}\right),\left(\mathscr{A}, w_{0}\right) \vDash \phi$ for all $w_{0} \in W$. 
COROLLARY 3. (ㅁ) $T_{2} \cup\{\square(\exists x) E x\} \vdash \phi$ iff for all structures, $\mathscr{A}=$ $(W, A, V)$ for $L\left(C_{0}\right)$ such that for all $w, w^{\prime} \in W A(w) \cap A\left(w^{\prime}\right)$ is non-empty, $\left(\mathscr{A}, w_{0}\right) \vDash \phi$ for all $w_{0} \in W$.

\section{INEXPRESSIBILITY RESULTS}

We first consider three examples.

(1) A structure $\mathscr{A}=(W, A, V)$ for $L\left(C_{0}\right)$ so that $(\mathscr{A}, w) \vDash T_{0}$ but $A(w) \neq$ $\bar{A}$ for all $w \in W$.

Let $W$ be the set of integers; select $A$ so that all $i \in W: A(i) \nsubseteq A(i+1)$, $A(i)$ is infinite, $\operatorname{card}(A(i+1))=\operatorname{card}(A(i)), \operatorname{card}(A(i+1)-A(i))$ is constant as $i$ varies; suppose $V^{\prime \prime} C_{0} \subseteq \cap\{A(i) \mid i \in W\}$ and for $P \in$ Pred, $V(w, P)$ is empty or $=f$ for all $w \in W$. Select $w \in W$ and $a_{1}, \ldots, a_{m} \in A(w)$. Select an automorphism $\sigma$ on $\bar{A}$ such that for all $i \in W, \sigma^{\prime \prime} A(i)=A(i+1)$ and $\sigma$ is constant on $V^{\prime \prime} C_{0} \cup\left\{a_{1}, \ldots, a_{m}\right\}$. For any $b_{1}, \ldots, b_{k} \in \bar{A}$, and $\phi$ a formula of $L\left(C_{0}\right)$,

$$
(\mathscr{A}, w) \vDash \phi\left[b_{1}, \ldots, b_{k}\right] \text { iff }(\mathscr{A}, w+1) \vDash \phi\left[\sigma\left(b_{1}\right), \ldots, \sigma\left(b_{k}\right)\right] .
$$

Suppose $(\mathscr{A}, w) \vDash \phi\left[a_{1}, \ldots, a_{m}\right]$. Given any $a_{m+1}, \ldots, a_{m+n} \in \bar{A}$ select a $j \in \omega$ so that $a_{m+1}, \ldots, a_{m+n} \in A(w+j)$. Then $(\mathscr{A}, w+j) \vDash$ $\phi\left[a_{1}, \ldots, a_{m}\right]$ and $(\mathscr{A}, w+j) \vDash\left(E x_{1} \& \ldots \& E x_{n}\right)\left[a_{m+1}, \ldots, a_{m+n}\right]$. Thus $(\mathscr{A}, w) \vDash T_{0}$.

(2) A structure $\mathscr{A}=(W, A, V)$ for $L\left(C_{0}\right)$ so that for $w_{0}, w_{1} \in W$, $A\left(w_{0}\right) \subsetneq A\left(w_{1}\right)$ and $\left(\mathscr{A}, w_{0}\right) \vDash(\square) T_{1}$. First we go on an algebraic digression.

Suppose $A \subseteq \bar{A}, A$ and $\bar{A}-A$ are countably infinite. For $F \subseteq A$, $G \subseteq \bar{A}-A, F$ and $G$ finite, let $A(F, G)=(A-F) \cup G$.

LEMMA. If $\sigma$ is an automorphism of $\bar{A}$ and $\sigma^{\prime \prime} A=A\left(F_{0}, G_{0}\right)$ then for any $F$ and $G, \sigma^{\prime \prime} A(F, G)=A\left(F^{\prime}, G^{\prime}\right)$ for $F^{\prime}=\left(\sigma^{\prime \prime} F \cap A\right) \cup\left(F_{0}-\sigma^{\prime \prime} G\right), G^{\prime}=$ $\left(\sigma^{\prime \prime} G-A\right) \cup\left(G_{0}-\sigma^{\prime \prime} F\right)$.

Suppose $\sigma$ is as in the antecedent. Consider $a \in A(F, G)$. We show that $\sigma(a) \in A\left(F^{\prime}, G^{\prime}\right)$. Case 1: $a \in A-F$. Then $\sigma(a) \in A\left(F_{0}, G_{0}\right)$. If $\sigma(a) \in$ $A-F_{0}, \sigma(a) \notin F^{\prime}$; so $\sigma(a) \in A\left(F^{\prime}, G^{\prime}\right)$. If $\sigma(a) \in G_{0}, \sigma(a) \in\left(G_{0}-\sigma^{\prime \prime} F\right)$; so $\sigma(a) \in G^{\prime}$, and so $\sigma(a) \in A\left(F^{\prime}, G^{\prime}\right)$. Case 2: $a \in G$. Then $\sigma(a) \notin$ $A\left(F_{0}, G_{0}\right)$. If $\sigma(a) \notin A$ and $\notin G_{0}, \sigma(a) \in\left(\sigma^{\prime \prime} G-A\right)$; so $\sigma(a) \in G^{\prime}$; so 
$\sigma(a) \in A\left(F^{\prime}, G^{\prime}\right)$. If $\sigma(a) \in F_{0}, \sigma(a) \in A$. Since $a \notin F, \sigma(a) \notin \sigma^{\prime \prime} F$; since $\sigma(a) \in \sigma^{\prime \prime} G, \sigma(a) \notin F^{\prime}$; so $\sigma(a) \in A\left(F^{\prime}, G^{\prime}\right)$. We next show that if $a \notin A(F, G)$ then $\sigma(a) \notin A\left(F^{\prime}, G^{\prime}\right)$. Case 1: $a \notin A$ and $a \notin G$. Then $\sigma(a) \notin A\left(F_{0}, G_{0}\right)$. If $\sigma(a) \notin A$ and $\notin G_{0}$, since $\sigma(a) \notin \sigma^{\prime \prime} G, \sigma(a) \notin G^{\prime}$; so $\sigma(a) \notin A\left(F^{\prime}, G^{\prime}\right)$. If $\sigma(a) \in F_{0}, \sigma(a) \in F_{0}-\sigma^{\prime \prime} G$; so $\sigma(a) \in F^{\prime}$; so $\sigma(a) \notin A\left(F^{\prime}, G^{\prime}\right)$. Case $2: a \in F$. Then $\sigma(a) \in A\left(F_{0}, G_{0}\right)$. If $\sigma(a) \in A-F_{0}, \sigma(a) \in \sigma^{\prime \prime} F \cap A$; so $\sigma(a) \in F^{\prime}$; so $\sigma(a) \notin A\left(F^{\prime}, G^{\prime}\right)$. If $\sigma(a) \in G_{0}, \sigma(a) \notin A ;$ since $\sigma(a) \notin \sigma^{\prime \prime} G ; \sigma(a) \notin G^{\prime}$; so finally $\sigma(a) \notin A\left(F^{\prime}, G^{\prime}\right)$.

Q.E.D.

For $\bar{A}$ and $A$ as above, let us introduce a constant $w(F, G)$ for every $F \subseteq A, G \subseteq \bar{A}-A, F$ and $G$ finite, so that $w(F, G)=w\left(F^{\prime}, G^{\prime}\right)$ iff $F=F^{\prime}$ and $G=G^{\prime}$; let $W=\{w(F, G) \mid$ for all such $F$ and $G\} \cup\left\{w_{1}\right\}$. Let $A(w(F, G))=A(F, G), A\left(w_{1}\right)=\bar{A}$. Select $V$, and $(W, A)$-valuation for $\left(C_{0}\right)$ so that where $P \in$ Pred, $V(w, P)$ is empty or $=f$ for all $w \in W$, and so that $A-V^{\prime \prime} C_{0}$ and $\bar{A}-\left(A \cup V^{\prime \prime} C_{0}\right)$ are infinite.

Suppose $\sigma$ is an automorphism of $\bar{A}, w, w^{\prime} \in W-\left\{w_{1}\right\}, \sigma^{\prime \prime} A(w)=A\left(w^{\prime}\right)$ and $\sigma$ is constant on $V^{\prime \prime} C_{0}$. Then $\sigma: \mathscr{A} \simeq \mathscr{A}$. (See [1], p. 202 for definition of $\sigma: \mathscr{A} \simeq \mathscr{A}$.)

Proof. Without loss of generality, we may suppose that $A(w)=A$. By the previous lemma for any $u \in W$ there is a $v \in W$ so that $\sigma^{\prime \prime} A(u)=A(v)$. Since $\sigma$ is constant on $V^{\prime \prime} C_{0}$, our claim follows.

LEMMA. Suppose $a_{1}, \ldots, a_{n} \in A(w), w \in W-\left\{w_{1}\right\}, \mathbf{a}=\left(a_{1}, \ldots, a_{n}\right)$. Then $(\mathscr{A}, w) \vDash \psi[\mathbf{a}]$ iff $\left(\mathscr{A}, w_{1}\right) \models \psi[\mathbf{a}]$.

Proof. Letting $w_{0}=w(\{\},\{\})$, we may assume without loss of generality, that $w=w_{0}$. Select a function $\rho: A \rightarrow \bar{A}, 1-1$, onto $\bar{A}$, and constant on $V^{\prime \prime} C_{0} \cup\left\{a_{1}, \ldots, a_{n}\right\}$. Clearly there is such a $\rho$. Suppose $\rho^{\prime} \subseteq \rho$, $\rho^{\prime}$ is finite. Let $F$ be the empty set, $G=\operatorname{range}\left(\rho^{\prime}\right)-A$. Then there is an automorphism $\sigma$ of $\bar{A}, \sigma \supseteq \rho^{\prime}$, so that $\sigma^{\prime \prime} A=A(F, G)$. By a previous remark, $\sigma: \mathscr{A} \simeq \mathscr{A}$. We may now apply Lemma 1 of [1] and conclude that $\left(\mathscr{A}, w_{0}\right) \vDash \psi[\mathbf{a}]$ iff $\left(\mathscr{A}, w_{1}\right) \vDash \psi\left[\rho\left(a_{1}\right), \ldots, \rho\left(a_{n}\right)\right]$; since $\rho$ is constant on $\left\{a_{1}, \ldots, a_{n}\right\}$, our result follows.

LEMMA. For any $w \in W,(\mathscr{A}, w) \vDash(\square) T_{1}$. To show this, suppose $a_{1}, \ldots, a_{n} \in \bar{A}, \mathrm{a}=\left(a_{1}, \ldots, a_{n}\right)$, and

$$
(\mathscr{A}, w) \mid=(\diamond \phi \& \diamond \psi \& \square\urcorner(\phi \& \psi))[\mathrm{a}] .
$$


Then for some $u, v \in W,(\mathscr{A}, u) \vDash \phi[\mathrm{a}],(\mathscr{A}, v) \vDash \psi[\mathrm{a}], u \neq v$. We wish to show that

$$
(\mathscr{A}, w) \vDash \diamond(\phi \&(\exists x) \diamond(\psi \& \neg E x))[\mathrm{a}] .
$$

If $u=w_{1}$ this is clear. Suppose $u \neq w_{1}$. By the previous lemma, we may select $v$ so that $v \neq w_{1}$. If $A(u)$ is not a subset of $A(v)$, we're done. Select $b \notin V^{\prime \prime} C_{0} \cup\left\{a_{1}, \ldots, a_{n}\right\}, b \notin A(v)$, and let $A\left(u^{\prime}\right)=A(u) \cup\{b\}$. Select an automorphism $\sigma$ of $\bar{A}$ constant on $V^{\prime \prime} C_{0} \cup\left\{a_{1}, \ldots, a_{n}\right\}$ and so that $\sigma^{\prime \prime} A(u)=$ $A\left(u^{\prime}\right)$. By previous remarks, $\sigma: \mathscr{A} \simeq \mathscr{A}$; so $(\mathscr{A}, u) \vDash \phi[\mathrm{a}]$ iff $\left(\mathscr{A}, u^{\prime}\right) \vDash \phi[\mathrm{a}]$. The lemma follows. Hence $\mathscr{A}$ and $w_{0}$ are as desired.

(3) A structure $\mathscr{A}=(W, A, V)$ for $L\left(C_{0}\right)$ so that for some $w_{0}, w_{1} \in W$, $A\left(w_{0}\right)$ and $A\left(w_{1}\right)$ are disjoint, but $\left(\mathscr{A}, w_{0}\right) \vDash(\square) T_{2} \cup\{\square(\exists x) E x\}$. Select $A\left(w_{0}\right)$ and $A\left(w_{1}\right)$ to be disjoint countable sets. For each $b \in A\left(w_{0}\right)$ and $c \in A\left(w_{1}\right)$ introduce distinct worlds $w_{0}(b, c)$ and $w_{1}(b, c)$, letting $A\left(w_{0}(b, c)\right)=A\left(w_{0}\right)-\{b\} \cup\{c\}, A\left(w_{1}\right)-\{c\} \cup\{b\}, W=\left\{w_{0}, w_{1}\right\} \cup$ $\left\{w_{0}(b, c), w_{1}(b, c) \mid b \in A\left(w_{0}\right), c \in A\left(w_{1}\right)\right\}$. Make sure that $A\left(w_{0}\right)-V^{\prime \prime} C_{0}$ and $A\left(w_{1}\right)-V^{\prime \prime} C_{0}$ are infinite; and let $V(w, P)$ be empty or $=f$ for all $w \in W, P \in$ Pred. For $\mathbf{a}=\left(a_{1}, \ldots, a_{n}\right), a_{i} \in \bar{A}=A\left(w_{0}\right) \cup A\left(w_{1}\right)$, if $b, c \notin\left\{a_{1}, \ldots, a_{n}\right\} \cup V^{\prime \prime} C_{0},\left(\mathscr{A}, w_{i}\right)=\phi[\mathrm{a}]$ iff $\left(\mathscr{A}, w_{i}(b, c)\right) \vDash \phi[\mathrm{a}]$. This is because of an automorphism $\sigma$ on $\bar{A}$ which fixes $\left\{a_{1}, \ldots, a_{n}\right\} \cup V^{\prime \prime} C_{0}$ and exchanges $b$ and $c$. Using this fact, we easily show that $\mathscr{A}$ is as desired.

We now consider various structural properties of frames $(W, A)$ and pairs of the form $\left((W, A), w_{0}\right), w_{0} \in W$.

PROPERTY 1. $\quad \bar{A}=A\left(w_{0}\right)$.

PROPERTY $\diamond 1$. For some $w \in W, \bar{A}=A(w)$.

PROPERTY 2. For some $w \in W, A\left(w_{0}\right) \subsetneq A(w)$.

PROPERTY $\square 2$. For each $w \in W$, there is a $w^{\prime} \in W$ so that $A(w) \subsetneq A\left(w^{\prime}\right)$.

PROPERTY $\diamond 2$. For some $w$ and $w^{\prime} \in W, A(w) \subsetneq A\left(w^{\prime}\right)$.

PROPERTY 3. For some $w \in W, A(w) \subsetneq A\left(w_{0}\right)$.

PROPERTY $\square 3$. For every $w \in W$, there is a $w^{\prime} \in W$ with $A\left(w^{\prime}\right) \subsetneq A(w)$. 
PROPERTY 4. For all $w \in W, A(w) \cap A\left(w_{0}\right)$ is non-empty.

PROPERTY 04 . For all $w$ and $w^{\prime} \in W, A(w) \cap A\left(w^{\prime}\right)$ is non-empty.

PROPERTY $\diamond 4$. For some $w \in W$ : for all $w^{\prime} \in W, A(w) \cap A\left(w^{\prime}\right)$ is non-empty.

PROPERTY 5. For some $w \in W, A(w)$ and $A\left(w_{0}\right)$ are disjoint.

PROPERTY $\square$ 5. For each $w \in W$, there is a $w^{\prime} \in W$ with $A\left(w^{\prime}\right)$ disjoint from $A(w)$.

PROPERTY $\diamond 5$. For some $w$ and $w^{\prime} \in W, A(w)$ and $A\left(w^{\prime}\right)$ are disjoint.

A structural property of frames $(W, A)$ for $w_{0} \in W$, is expressible in $L\left(C_{0}\right)$ iff for some set $T$ of sentences of $L\left(C_{0}\right)$ : for all structures $\mathscr{A}=(W, A, V)$ and $w_{0} \in W,\left(\mathscr{A}, w_{0}\right)=T$ iff $(W, A)$ has that property. A similar definition applies to properties of pairs $\left((W, A), w_{0}\right)$, where $(W, A)$ is a frame and $w_{0} \in W$.

THEOREM 4. The negation of Property 1 is unexpressible. This is equivalent to saying “There could be something which doesn't acutally exist" is unexpressible. Suppose that for $T$ a set of sentences of $L\left(C_{0}\right)$, if $\bar{A} \neq A\left(w_{0}\right)$ then $\left((W, A, V), w_{0}\right) \vDash T$. Example 1 shows that $T \cup T_{0}$ is consistent; then Theorem 1 yields a structure $\mathscr{A}=(W, A, V)$ and $w_{0} \in W$ so that $(\mathscr{A}, w) \vDash T$ but $\bar{A}=A\left(w_{0}\right)$. (Kit Fine has pointed out to me that this theorem follows painlessly from examination of the models described at the bottom of p. 203 of [1].)

THEOREM 5. Property 1 is unexpressible. This is equivalent to saying that "Necessarily everything actually exists" is unexpressible. Suppose that $T$ is a set of sentences of $L\left(C_{0}\right)$ and that for any structure $\mathscr{A}=(W, A, V)$ for $L\left(C_{0}\right)$ and $w_{0} \in W$, if $\left(\mathscr{A}, w_{0}\right) \models T$ then $\left((W, A), w_{0}\right)$ has Property 1. For $\mathscr{A}$ as in Example 1, $(\mathscr{A}, i) \mid \neq T$; select $\phi \in T$ so that $(\mathscr{A}, i) \mid \neg \phi$; then $T_{0} \cup\{\neg \phi\}$ is consistent; so Theorem 1 yields a structure $\mathscr{B}=\left(U, B, V^{\prime}\right)$ for $L\left(C_{0}\right)$ and $u_{0} \in U$ so that $\left.\left(\mathscr{B}, u_{0}\right)=\right\urcorner \phi$, so $\left(\mathscr{B}, u_{0}\right) \not \neq T$, but $\left((U, B), u_{0}\right)$ has Property 1. 
THEOREM 6. The negation of Property $\diamond 1$ is unexpressible. Identifying the proposition expressed by a sentence in a structure with the set of worlds at which that sentence is true, this tells us that the necessitation of the proposition expressed by "There could be something which doesn't actually exist" is unexpressible. Notice: that proposition is not expressed by "Necessary there could be something which doesn't actually exist", because of the way in which "actually" refers back to the "starting world". Suppose that for any structure $\mathscr{A}=(W, A, V)$ for $L\left(C_{0}\right)$, if $(W, A)$ does not have Property $\diamond 1$, then $\left(\mathscr{A}, w_{0}\right) \vDash T$ for $w_{0} \in W$. By examining Example 1, $T \cup T_{0}$ is consistent. Theorem 1 then delivers a structure $\mathscr{A}=(W, A, V)$ for $L\left(C_{0}\right)$ so that for some $w_{0} \in W,\left(\mathscr{A}, w_{0}\right) \vDash T$ and $(W, A)$ has Property $\diamond 1$.

Curiously, we can express the necessitation of the proposition expressed by "Necessarily everything actually exists" by " $\square(\forall x) \square E x$ "; of course, “ $(\forall x) \square E x$ ” doesn't express “Necessarily everything actually exists". So the necessitation of an unexpressible proposition may be expressible.

THEOREM 7. Property $\diamond 1$ is unexpressible. This shows the possibilification of the proposition "Necessarily everything actually exists", is unexpressible. Suppose that for some $T$, if $\mathscr{A}=(W, A, V)$ and $\left(\mathscr{A}, w_{0}\right) \vDash T$ then $(W, A)$ has Property $\diamond 1$. For $\mathscr{A}$ of Example $1,(\mathscr{A}, i) \not T$; say $(\mathscr{A}, i) \vDash \neg \phi$ for $\phi \in T$. The argument is as for Theorem 5 .

Again, the possibilification of the proposition expressed by "There could be something which doesn't actually exist" is expressible by " $\diamond(\exists x) \diamond E x$ ".

THEOREM 8. Property 2 is unexpressible. This shows the unexpressibility of "There could be something which doesn't actually exist without there not being something which does actually exist". Suppose for any structure $\mathscr{A}=(W, A, V)$ for $L\left(C_{0}\right)$ and $w_{0} \in W$, if $\left((W, A), w_{0}\right)$ has Property 2 then $\left(\mathscr{A}, w_{0}\right) \vDash T$. Example 1 shows that $T \cup T_{0}$ is consistent; Theorem 1 delivers a structure $\mathscr{A}=(W, A, V)$ and $w_{0} \in W$ so that $\left(\mathscr{A}, w_{0}\right) \vDash T$ but $\left((W, A), w_{0}\right)$ lacks Property 2.

THEOREM 9. Property $\square 2$ is unexpressible. Proof is as above.

THEOREM 10. Property $\square 3$ is unexpressible. Proof is as above.

THEOREM 11. Property $\diamond 2$ is unexpressible. Suppose we have $T$ so that 
for any structure $\mathscr{A}=(W, A, V)$ and $w_{0} \in W$, if $(W, A)$ has Property $\diamond 2$ then $\left(\mathscr{A}, w_{0}\right) \vDash T$. Consideration of Example 2 shows that $T \cup(\square) T_{1} \cup$ $\{\square(\exists x) E x\}$ is consistent; Theorem 2 yields a structure $\mathscr{A}=(W, A, V)$ so that $\left(\mathscr{A}, w_{0}\right)=T$ but $(W, A)$ lacks Property $\diamond 2$.

THEOREM 12. Property 3 is unexpressible. Proof is as above.

THEOREM 13. Property 4 is unexpressible. This says that "Necessarily something actual exists" is unexpressible. Suppose we have $T$ so that if $\mathscr{A}=(W, A, V),\left(\mathscr{A}, w_{0}\right)=T$, then $\left((W, A), w_{0}\right)$ has Property 4 . For the $\mathscr{A}$ of Example 3, $\left((W, A), w_{0}\right)$ does not have Property 4; so $\left(\mathscr{A}, w_{0}\right) \not \# T$; select $\phi \in T$ so that $\left(\mathscr{A}, w_{0}\right) \vDash \neg \phi ;\{\neg \phi\} \cup(\square) T_{2} \cup\{\square(\exists x) E x\}$ is consistent; let $\mathscr{B}=\left(U, B, V^{\prime}\right)$ and $u_{0} \in U$ be delivered by Theorem $3 ;\left(\mathscr{B}, u_{0}\right) \vDash \neg \phi$, so $\left(\mathscr{B}, u_{0}\right) \not \neq T$, but $\left((u, B), u_{0}\right)$ has Property 4 .

THEOREM 14. Property 04 is unexpressible. Proof is as above.

THEOREM 15. Property 5 is unexpressible.

THEOREM 16. Property $\square 5$ is unexpressible.

THEOREM 17. Property $\diamond 4$ is unexpressible.

THEOREM 18. Property $\diamond 5$ is unexpressible.

Proofs should now be routine.

Conjecture. There is no set $T$ of sentences of $L\left(C_{0}\right)$ so that for all structures $\mathscr{A}=(W, A, V)$ for $L\left(C_{0}\right)$ and $w_{0} \in W$ :

$$
\begin{gathered}
\left(\mathscr{A}, w_{0}\right)=T \text { iff for all } w \in W, \text { if } A\left(w_{0}\right) \neq A(w) \text { then } \\
\qquad A\left(w_{0}\right) \cap A(w) \text { is empty. }
\end{gathered}
$$

It should be noted that $\left(\mathscr{A}, w_{0}\right) \vDash \phi$ iff for all $w, w^{\prime} \in W$, if $A(w) \neq$ $A\left(w^{\prime}\right)$ then $A(w) \cap A\left(w^{\prime}\right)$ is empty, where $\phi$ is:

$$
\square(\forall x) \square(\forall y)(\diamond(E x \& \neg E y) \supset \square(E x \supset(\forall z) \square(E y \supset \neg E z)) .
$$

Observation. If we consider logics other than S5, and permit corresponding accessibility relations to occur in frames, these inexpressibility results 
carry over to the resulting sort of structures. These results are really just weakenings of the preceding ones. It would be nice to have a single general theorem which explains exactly what distinguishes the expressible from the unexpressible properties.

When we move to a second-order modal language, we can express, for example, "There could be something which doesn't actually exist":

$$
(\exists X)(\neg(\exists x) X x \& \diamond(\exists x)(X x \& \square X x)) .
$$

Note added in proof (Sept. 30, 1983): In fact, all properties discussed above are expressible in the second-order language in which type 1 variables range over essences (i.e. over subsets of $\bar{A}$ ), with Pred and $C$ empty. This is discussed further in 'On modal logics which enrich first-order S5', forthcoming in this Journal.

\section{REFERENCES}

[1] Kit Fine, 'Failures of the interpolation lemma in quantified modal logic', Journal of Symbolic Logic 44, No. 2 (1979).

[2] Kit Fine, 'Model theory of modal logic, I', Journal of Philosophical Logic 7 (1978), 125-156.

[3] Allen Hazen, 'Expressive completeness in modal languages', Journal of Philosophical Logic 5 (1976), 25-46.

Sage School of Philosophy,

Cornell University,

Ithaca,

NY 14853, U.S.A. 